\title{
Síndrome de Tolosa-Hunt, un diagnóstico de exclusión
}

Publicado en Internet:

13-marzo-2015

María del Pilar Martín-Tamayo Blázquez: mpmtamayo@hotmail.com
Palabras clave:

- Síndrome

de Tolosa-Hunt

- Oftalmoplejia dolorosa

\section{I. Buedo Rubio ${ }^{a}$, M. P. Martín-Tamayo Blázquez ${ }^{b}$, I. Onsurbe Ramírez $^{b}$}

${ }^{a}$ Servicio de Pediatría. Complejo Hospitalario Universitario de Albacete. Albacete. España • bunidad de Neuropediatría. Servicio de Pediatría. Complejo Hospitalario Universitario de Albacete.

Albacete. España.

\section{Tolosa-Hunt syndrome, a diagnosis of exclusion}

La oftalmoplejia dolorosa es una entidad infrecuente en Pediatría, caracterizada por dolor orbitario y parálisis oculomotora ipsilateral. Puede ser debido a diferentes causas; una de ellas es el síndrome de Tolosa-Hunt (STH), causado por inflamación inespecífica en el seno cavernoso o fisura orbitaria superior. El STH es un diagnóstico de exclusión, por lo que ante un paciente con sospecha hay que realizar los pertinentes exámenes complementarios, que incluyen pruebas de imagen cerebral, entre otras. El STH suele presentar buena evolución y respuesta a tratamiento corticoideo aunque en ocasiones puede persistir la oftalmoplejia en diferentes grados y la recurrencia no es infrecuente. Painful ophthalmoplegia is a rare entity in children, characterized by orbital pain and ipsilateral oculo-
motor paresis. The Tolosa-Hunt syndrome (THS), is caused by a granulomatous inflammation involving

Key words:

- Tolosa-Hunt the cavernous sinus or superior orbital fissure of unknown etiology.

syndrome

The diagnosis of THS is based upon the clinical presentation in conjunction with neuroimaging results. The STH usually has good clinical response to corticosteroids, but occasionally ophthalmoplegia persists

- Painful opthalmoplegia in varying degrees and some patients follow a relapsing-remitting course.

\section{INTRODUCCIÓN}

La oftalmoplejia dolorosa es una entidad infrecuente en Pediatría. Se caracteriza por dolor orbitario acompañado de parálisis oculomotora ipsilateral, con o sin parálisis simpática ocular y pérdida sensitiva en la distribución de la rama oftálmica o maxilar del trigémino. Existen diferentes causas que pueden producirlo, dentro de las cuales se en- cuentra el síndrome paraselar, el síndrome orbitario y otras, como la migraña oftalmopléjica.

Dentro de las causas paraselares destaca el síndrome de Tolosa-Hunt, patología poco frecuente, caracterizada por dolor periorbitario unilateral asociado a lesión ipsilateral de los pares craneales III, IV, VI y/O primera y segunda rama del $\mathrm{V}$, como consecuencia de inflamación inespecífica a nivel del seno cavernoso, fisura orbitaria superior o ápex orbitario. 


\section{CASO CLÍNICO}

Se presenta el caso de una niña de siete años que acude al Servicio de Urgencias por ptosis palpebral derecha y dolor periorbitario de 24 horas de evolución, que no cede con analgésicos habituales, encontrándose afebril, con nauseas y vómitos esporádicos y sin proceso infeccioso intercurrente.

La paciente no presentaba antecedentes personales ni familiares de interés, salvo un episodio similar al descrito en los dos años previos, con una duración de dos semanas, que cedió espontáneamente sin medicación. En la exploración física se objetivaba una parálisis de la supraducción y de la aducción de la mirada del ojo derecho, con ptosis palpebral, sin afectación pupilar ni alteración de otros pares craneales (Fig. 1). Se realizó un hemograma y una bioquímica general, velocidad de sedimentación globular, proteína $\mathrm{C}$ reactiva, hemoglobina glicosilada, anticuerpos anti-ADN, anticuerpos antinucleares (ANA), anticuerpos extraíbles del núcleo (ENA), anticuerpos anticitoplasma de neutrófilos (ANCA), y resonancia magnética craneal, siendo todos los resultados normales. Se diagnosticó síndrome de Tolosa-Hunt y se pautó tratamiento oral con prednisona $1 \mathrm{mg} / \mathrm{kg} /$ día durante cinco días. En las siguientes 48 horas se reevaluó a la paciente, que presentó una resolución completa de la oftalmoplejia y del dolor, con persistencia de ptosis palpebral leve, que se mantuvo durante los siguientes seis meses.

\section{DISCUSIÓN}

El síndrome de oftalmoplejia dolorosa consiste en un cuadro de dolor periorbitario o hemicraneal, combinado con parálisis ipsilateral de los nervios oculomotores (III, IV, VI) y en ocasiones parálisis del sistema nervioso simpático y/o pérdida de sensibilidad en la distribución de la rama oftálmica y/o maxilar del nervio trigémino ${ }^{1,2}$. Los tres nervios oculomotores pueden estar alterados en varias combinaciones, sin embargo, no suele aparecer afectación pupilar ${ }^{1}$. En nuestro caso se produjo
Figura 1. Se aprecia la ptosis palpebral derecha junto con afectación de la supraducción y de la aducción de la mirada

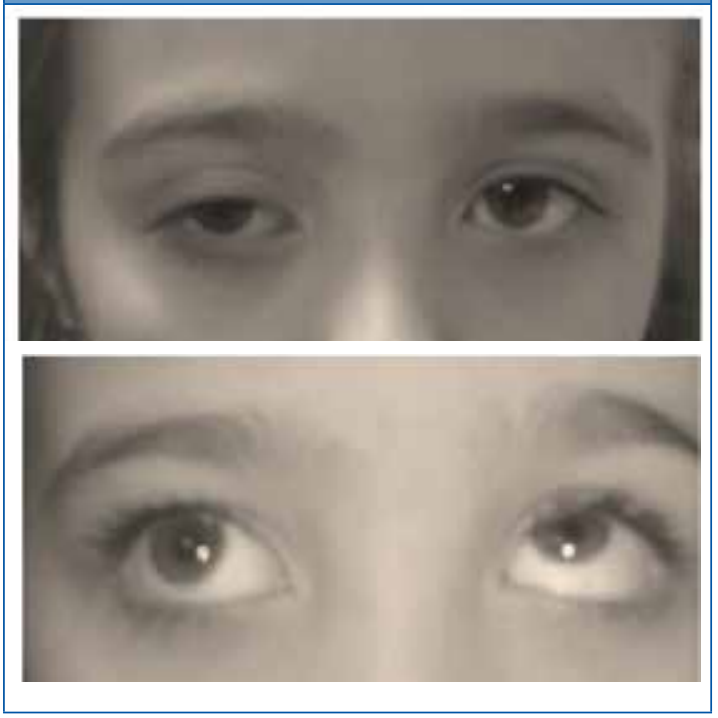

parálisis aislada del III par craneal sin afectación pupilar, no presentando alteraciones en otros pares craneales. Tampoco mostraba afectación de la visión, aunque en algunos pacientes puede aparecer disfunción del nervio óptico, indicando afectación del vértice de la órbita ${ }^{1}$. Por otro lado, no se han hallado casos con afectación sistémica, salvo nauseas y vómitos ${ }^{1}$, también presentes en nuestra paciente.

Tolosa describió en $1954^{3}$ el caso de un paciente con dolor ocular izquierdo, junto con pérdida visual, oftalmoplejia completa e hipoestesia en la primera rama del trigémino. Posteriormente, Hunt ${ }^{4}$ describió seis casos similares en los que observó buena respuesta clínica con terapia corticoidea y la posibilidad de remisiones espontáneas, así como de recurrencias ${ }^{5}$. El primer paciente descrito presentó en la necropsia una inflamación granulomatosa en el seno cavernoso, pero en otros pacientes la exploración quirúrgica de esta región no ha evidenciado alteraciones patológicas ${ }^{6}$. Así, aunque a pesar de los estudios realizados la etiología no está claramente establecida, se admite que es secundario a un proceso inflamatorio inespecífico en el seno cavernoso, fisura orbitaria superior o ápex orbitario $^{5}$ 
En cuanto a los datos epidemiológicos, no se ha demostrado que exista diferencia en la proporción de hombres y mujeres afectados, pudiendo presentarse en personas de cualquier edad, siendo muy infrecuente en menores de 20 años $^{6}$.

El diagnóstico de síndrome de Tolosa-Hunt requiere un estudio exhaustivo que permita descartar patología vascular, traumática, tumoral e infeccio$\mathrm{sa}^{3}$. Siguiendo los criterios diagnósticos que se han establecido por la International Headache Society (Tabla 1) ${ }^{1,2,5}$, la presencia de dolor orbitario unilateral y oftalmoplejia junto con la buena respuesta al tratamiento corticoideo, una vez excluidas las patologías anteriores, nos permiten establecer el diagnóstico, a pesar de la ausencia de signos inflamatorios en la resonancia magnética.

En nuestro caso, la concordancia clínica, la espectacular respuesta al tratamiento con corticoides en 48 horas y la exclusión de otras posibles entidades causantes de oftalmoplejia dolorosa mediante los pertinentes estudios analíticos y de neuroimagen, permitieron establecer el diagnóstico de síndrome de Tolosa-Hunt.

La remisión espontánea es frecuente, incluso en aquellos casos en los que no se instaura tratamiento con corticoides. Nuestra paciente presentaba un episodio previo con recuperación espontánea en dos semanas.

Sin embargo, aunque el pronóstico es bueno y la resolución del dolor es completa con o sin trata-

\section{BIBLIOGRAFÍA}

1. Kline LB, Hoyt WF. The Tolosa-Hunt sydrome. J Neurol Neurosurg Psychiatry. 2001;71:577-82.

2. Mora de Oñate J, Pascual Pérez Alfaro R, Izquierdo Vázquez C, Gónzalez Ruiz M, Aguirrebeña Olmos A, Díez Villalba R. Oftalmoplejía dolorosa (pesudotumor y sindrome de Tolosa Hunt). Arch Soc Esp Oftalmol. 2007;82:509-12.

3. Tolosa E. Periarteritic lesions of the carotid siphon with the clinical features of a carotid infraclinoidal aneurysm. J Neurol Neurosurg Psychiatry. 1954;11:56.
Tabla 1. Criterios diagnósticos de la International Headache Society para el síndrome de Tolosa-Hunt

- Uno o más episodios de dolor orbitario unilateral, que persiste semanas en ausencia de tratamiento

- Asocia parálisis del III, IV y VI par craneal, aislados o en conjunto, y/o demostración de granuloma en resonancia magnética cerebral o biopsia

- La oftalmoparesia coincide con el comienzo del dolor o le sigue en menos de dos semanas

- El dolor y la parálisis ceden en 72 horas cuando se instaura un correcto tratamiento corticoideo

- Se deben haber excluido otras lesiones causales mediante investigaciones adecuadas

miento, la recurrencia es frecuente en estos pacientes, sucede entre un 30-40\% de los $\operatorname{casos}^{6}, y$ puede ser ipsilateral, contralateral y bilateral, aunque de forma más inusual ${ }^{1}$. No obstante, pese a la buena evolución y a la resolución completa del dolor, en algunas ocasiones pueden persistir anomalías en la motilidad ocular o palpebral.

\section{CONFLICTO DE INTERESES}

Los autores declaran no presentar conflictos de intereses en relación con la preparación y publicación de este artículo.

\section{ABREVIATURAS}

ANA: anticuerpos antinucleares - ANCA: anticuerpos anticitoplasma de neutrófilos • ENA: anticuerpos extraíbles del núcleo • STH: síndrome de Tolosa-Hunt.

4. Hunt W, Meagher JN, Lefever H, Zenan W. Painful opthalmoplegia. Its relation to indolent inflammation of the cavernous sinus. Neurology. 1961;11: 56-62.

5. Hamad Cueto O, Tamayo Toledo JA, Mármol Prados AA, García Trujillo L, López Madrona JC, Fernández Fernández O. Síndrome de Tolosa-Hunt y pseudotumor orbitario. Entidades solapadas en un caso con perfil clínico no habitual. Rev Neurol. 2006;42:530-4.

6. Jiménez Caballero PE, Florensa J, Marsal Alonso C, Álvarez Tejerina A. Síndrome de Tolosa-Hunt de repetición con neuroimagen normal. Descripción de tres casos. Rev Neurol. 2005;41:30-3. 